\title{
LE JEU DES LANGUES \\ DANS LE PAYSAGE LINGUISTIQUE URBAIN AU MAROC
}

\section{EL JUEGO DE LAS LENGUAS EN EL PAISAJE LINGÜÍSTICO URBANO DE MARRUECOS}

\section{THE PLAY OF LANGUAGES IN THE URBAN LINGUISTIC LANDSCAPE IN MOROCCO}

\author{
لعبة اللغات في المشهد اللغوي الحضري بالمغرب \\ Leila MESSAOUDI* \\ Université Ibn Tofail (Kénitra, Maroc) \\ Mustapha KHIRI \\ Université Moulay Ismail (Meknès, Maroc)
}

BIBLID [1133-8571] 26 (2019) 02.1-25.

Résumé : A partir de deux approches, le paysage linguistique (Landry \& Bourhis 1997) et la sociolinguistique urbaine (Calvet 1994 et Bulot \& Messaoudi 2003), nous présentons les résultats d'un travail de terrain sur l'interaction des langues dans le paysage linguistique marocain à travers la collecte et l'étude de badges dans certains quartiers de Rabat et d'Errachidia. L'étude a montré que le choix des langues et des orthographes utilisées dans les insignes des deux villes est régi par de nombreux facteurs. Il convient de noter qu'une attention particulière est accordée aux domaines et aux rôles des variétés linguistiques dans ce jeu de langue, et notamment à la dariya (arabe marocain). Mots clés: Paysage linguistique, Jeu linguistique, Urbain, Ségrégation spatiale, Dariya, Arabe standard, Français, Amazigh, Rabat, Errachidia

E-mail: 1messaoudi@gmail.com. Laboratoire Langage et société-CNRST-URAC56.
E-mail: mkhiri@gmail.com. Laboratoire Langage et société-CNRST-URAC56. 
Resumen $^{(1)}$ : Desde dos enfoques, el del Paisaje lingüístico (linguisticlandscape, Landry\&Bourhis 1997) y el de la Sociolingüística urbana (Calvet 1994 y Bulot \& Messaoudi 2003), se presentan los resultados de un trabajo de campo sobre el juego de las lenguas en el paisaje lingüístico marroquí a través de la recopilación y el estudio de insignias en algunos barrios de Rabat y Errachidia. El estudio ha mostrado que la elección de lenguas y grafías en uso en las insignias de las dos ciudades está regida por muchos factores. Cabe señalar que se presta especial atención a los ámbitos y roles de las variedades lingüísticas en este juego de idiomas y, en particular, a la dariya (árabe marroquí).

Palabras clave: Paisaje lingüístico (landscape), Juego de lenguas, Urbano, Segregación espacial, Dariya, Árabe estándar, Francés, Amazigh, Rabat, Errachidia.

Abstract $^{(2)}$ : «The game of languages in the urban linguistic landscape in Morocco». This article presents, in the context of two approaches: the linguistic landscape (Landry \& Bourhis 1997) and urban sociolinguistics (Calvet 1994, Bulot and Messaoudi 2003), the findings of a field work study on the game of languages in the urban linguistic landscape in Morocco through the collection and study of signs in some districts of Rabat and Errachidia. The study showed that the choice of languages and graphics used in the signs of the two cities is governed by several factors. Particular attention is paid to the domains and roles of the linguistic varieties in this language game and in particular to Darija (Moroccan Arabic).

Key words: Linguistic landscape, Languages game, Urban, Spatial segregation, Darija, Standard Arabic, French, Amazigh, Rabat.

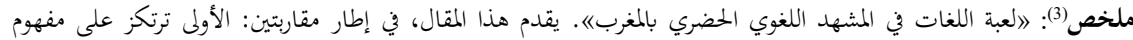

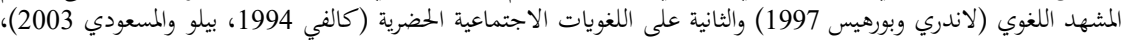

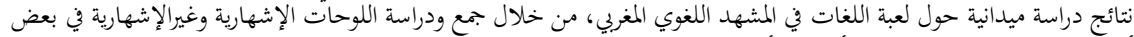

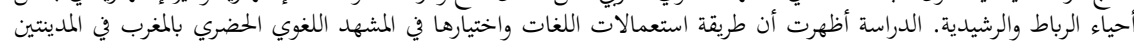

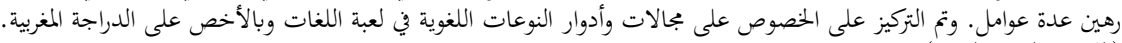
(اللهجة المغربية العربية)

الكلمات المفتاحة: لعبة اللغات، التمبيز المجالي، الدارجة، اللغة العربية الفصحى، الفرنسية، الأمازيغية، الرباط، الراشيدية، المشهد اللغوي الحضري.

(1) Nous remercions Mme Silvia Nieves Gómez Rodríguez (biblioteca municipal Vapor Badia de Sabadell.) et Lahcen Elbelghiti pour la traduction du résumé en espagnol.

(2) Nous remercions M. Abderrahman Babni (Enseignant chercheur à la Faculté Polydisciplinaire d'Errachidia) pour la traduction du résumé en anglais.

(3) Nous remercions M. Abdelmajid Talha (Enseignant chercheur à la faculté Polydisciplinaire d'Errachidia) pour son aide dans la traduction du résumé en arabe.

$A A M, 26(2019) 02.1-25$ 


\section{Introduction}

Le présent article se propose, dans le cadre général de la sociolinguistique, d'étudier le jeu des langues dans le paysage linguistique urbain au Maroc à partir des données d'un travail de terrain mené sur deux villes : Rabat et Errachidia dont la signalétique et le marquage spatial ont déjà fait l'objet de précédents travaux tels que ceux de Messaoudi \& Mzioud (2012) et de Khiri (2009).

Nous focaliserons notre attention sur deux volets. Un premier volet sera consacré à l'identification des variétés linguistiques en usage dans l'environnement graphique urbain, en opérant une distinction entre le secteur public (comprenant l'Etat et ses institutions) et celui du secteur du privé (englobant les banques, les entreprises, les commerces, etc.) et en s'appuyant sur quelques catégorisations selon les lieux d'enquête et les domaines

Un second volet sera réservé aux variétés employées dans un domaine précis, celui de la publicité.

Dans ces deux volets, seul le contenu verbal scripturaire sera retenu, en ignorant sciemment les aspects sémiotiques caractérisant l'environnement graphique urbain.

Auparavant, nous commencerons par présenter le cadre théorique et les aspects méthodologiques.

\section{Cadre théorique et aspects méthodologiques}

\subsection{Cadre théorique}

Cette étude se situe dans le cadre de la sociolinguistique urbaine, en mobilisant la notion de Linguistic Landscape définie par Landry \& Bourhis (1997: 25) comme suit: «the language of public road signs, advertising billboards, street names, place names, commercial shop signs, and public signs on government buildings combines to form the linguistic landscape of a given territory, region, or urban agglomeration» ${ }^{(4)}$.

La dénomination anglo-saxonne de Linguistic Landscape, rendue en français par l'expression «paysage linguistique» (Landry \& Bourhis, op. cit.)

(4) «La langue de l'ensemble des plaques de signalisation routière, des panneaux publicitaires, des noms de rue, des noms de places, des enseignes commerciales, des magasins commerciaux et des panneaux publics sur les édifices gouvernementaux constitue le paysage linguistique d'un territoire, d'une région ou d'une agglomération urbaine donnée ». Notre traduction 
n'est pas sans lien avec celle francophone de sociolinguistique urbaine $e^{(5)}$ comme le signalait Bulot $(2011: 6):$ «[...] les travaux - tant descriptifs que théoriques - relevant respectivement de la sociolinguistique urbaine et des Linguistic Landscape Studies, portent sur des espaces urbains ou pour le moins sur des espaces fondamentalement organisés et quasi-voués à la culture urbaine ».

Ceci étant et pour plus de précision sur le plan terminologique, il serait plus pertinent d'user de l'expression paysage linguistique urbain car celle de paysage linguistique peut prêter à confusion car elle peut s'appliquer à n'importe quel type de territoire, qu'il soit rural, urbain, urbanisé, rurbain, etc. ; et peut même être interprétée comme synonyme de situation linguistique.

Cette même remarque vaut, du reste, pour l'appellation anglophone comme ne manque pas de le signaler Durk Gorter (2006: 2): «Instead of calling linguistic landscape, it could also be named Linguistic Cityscape». Intéressant de noter que le mot City apparaît dans cette proposition, permettant ainsi de lever l'ambigüité.

\subsection{Aspects méthodologiques}

La méthode retenue pour aborder le terrain considéré est celle de l'enquête par observation anonyme.

L'enquête sur le paysage linguistique urbain au Maroc a été effectuée dans deux villes : Rabat, la capitale du pays, et Errachidia, petite ville située au Sudest. Elle a concerné quatre sites d'enquête correspondant à quatre quartiers : deux à Rabat (Hay Ryad et Akkari) et deux à Errachidia (Centre ville et Targa Jdida). Cette enquête s'est déroulée pendant des périodes espacées entre janvier et février 2019 et a permis la collecte d'un riche corpus.

Durant l'enquête, nous avons pris des photos d'enseignes, d'affiches, d'écriteaux, de panneaux et de plaques visibles et à la portée du public dans les quartiers, les avenues, les rues et les ronds-points des deux villes objets de l'étude.

Il est à noter qu'il nous a été impossible de couvrir l'ensemble des territoires des quatre quartiers des deux villes. A Hay Ryad, nous avons photographié les enseignes de l'avenue Ennakhil, artère principale du quartier, et de Mahaj Ryad son noyau huppé.

(5) L'on peut référer, à titre indicatif, aux travaux de sociolinguistique urbaine de Calvet (1994) et Bulot \& Messaoudi (2003) (voir bibliographie). 
Pour Akkari, nous avons pris en photo les enseignes dans l'ensemble du quartier, en nous focalisant sur l'avenue Assalam et Souiqua ${ }^{(6)}$, deux lieux animés de ce quartier populaire.

En ce qui concerne Errachidia, nous avons pris des photos des enseignes dans de différents endroits de la ville et notamment au centre et au quartier Targa Jdida.

A Rabat, la distinction est manifeste entre le quartier huppé (Hay Riad) et celui populaire (Akkari) ; elle est en revanche moins visible entre les quartiers d'Errachidia qui présentent une certaine homogénéité, sans distinction sociale frappante.

L'enquête s'est déroulée les matins ou les après-midis pour obtenir une bonne clarté des photos. Nous avons effectué, de manière quasi-systématique, une prise des photos des enseignes tout au long des lieux d'enquête. Nous nous étions dotés d'un appareil photo numérique qui permet la prise de photos à distance.

Nous n'avons pas eu besoin de demander à quiconque, chaque fois, l'autorisation de prendre des photos des enseignes. Nous l'avons fait, dans les cas où un obstacle naturel, le reflet des rayons du soleil, ou une barrière visuelle, un haut camion stationné, nous empêchait de faire notre travail.

Dans les cas où nous avons sollicité l'autorisation de prendre des photos des enseignes, les personnes que nous avons trouvées dans les locaux commerciaux concernés nous ont demandé les raisons de cette enquête. Nous leur avons répondu, en toute vérité, que c'est pour une étude sur les langues en usage dans les enseignes au Maroc. Après, elles nous ont accordé la permission d'effectuer des photos.

L'enquête s'est réalisée dans de bonnes conditions, mais il n'en demeure pas moins qu'à trois reprises, on nous a sommés d'arrêter de prendre des photos. A Mahaj Ryad, un agent de sécurité privée nous a conduits vers le patron d'un commerce franchisé qui, à son tour, nous a notifié qu'il faut disposer d'une autorisation officielle parce que, selon lui, la zone est hautement sécurisée. Au quartier Akkari, un gérant d'une station-essence a demandé d'éviter de photographier les prix affichés du carburant. A Errachidia, un agent de police, près de la gare routière, n'a demandé que de l'épargner et de prendre toutes les photos désirées. La prise des photos sur la voie publique n'est plus un tabou et ne

(6) Souiqua : nom féminin (diminutif de "souk" (marché) petit souk qui se tient chaque jour à la différence du souk qui est hebdomadaire 
suscite plus trop de méfiance comme c'était le cas avant l'adoption massive des Smartphones.

\subsection{Le corpus}

Le corpus recueilli dans les quartiers étudiés des deux villes (Rabat et Errachidia), compte 278 photos d'enseignes de la signalétique qui peut être définie comme suit : «(...) elle [la signalétique] est l'ensemble des traces écrites ou iconiques qui sont reçues par une population pour lui permettre de s'orienter dans un territoire donné» (Messaoudi \& Mzioud 2012 : 177).

En fait, le corpus comprend les enseignes commerciales ainsi que celles des administrations publiques et des cabinets privés ; les panneaux de signalisation routière et les noms de rues. 29.

Quant aux photos prises des affiches publicitaires, elles sont au nombre de

\subsection{Les affiches et enseignes de la signalétique}

Dans ce premier axe, nous identifierons les variétés linguistiques utilisées dans les enseignes collectées (à l'exception des enseignes/affiches publicitaires qui seront traitées dans l'axe 2). 


\subsubsection{Identification des variétés linguistiques et catégorisation des données} recueillies

D'un point de vue linguistique, les enseignes retenues pour cet axe sont, de par le contenu et la graphie, soit monolingues, soit bilingues, soit multilingues. Les variétés linguistiques mobilisées sont l'arabe (7), l'amazigh, le français, l'anglais et l'espagnol.

Fig. 1

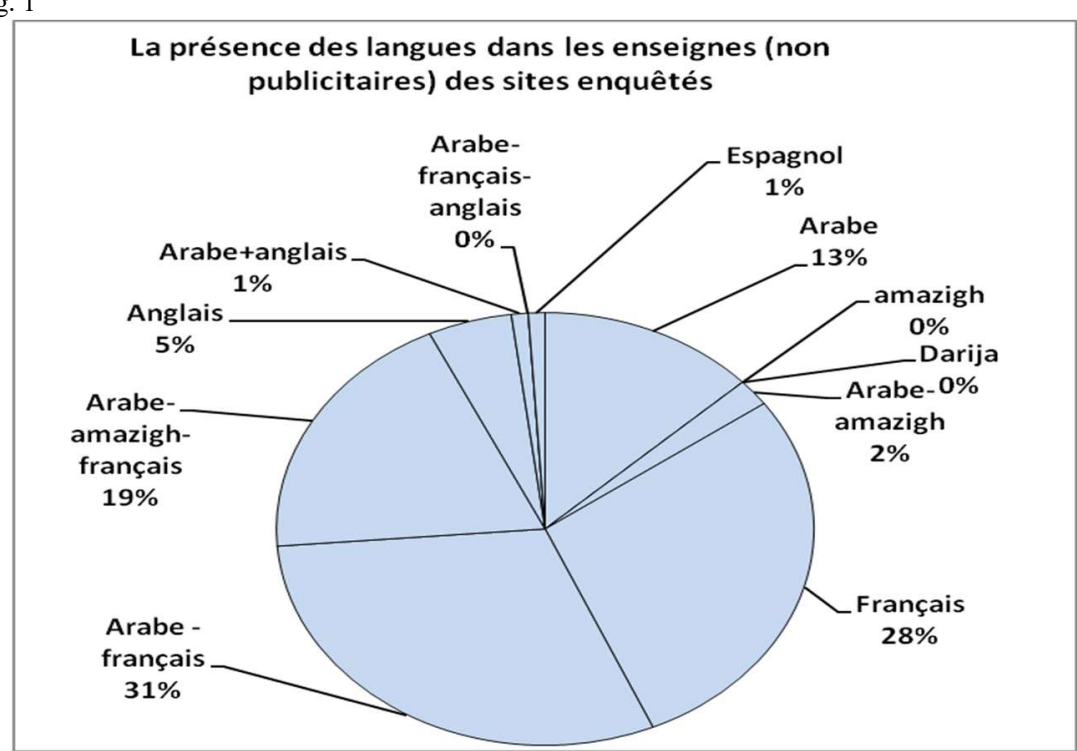

Le choix des langues utilisées dans ces enseignes diffère selon plusieurs facteurs : les lieux d'enquête (villes et quartiers); le type du secteur (public et privé) ; le domaine d'activité.

\subsubsection{Les enseignes selon les lieux d'enquête}

La collecte des photos des enseignes s'est faite dans deux villes différentes à plusieurs égards. Tous les indices de développement socio-économiques sont

(7) L'appellation «arabe» désigne dans ce travail l'arabe standard. Darija désigne l'arabe dialectal marocain, variété arabe véhiculaire au Maroc. 
nettement en faveur de Rabat. A Errachidia, il n'existe aucun quartier à comparer à Hay Ryad. Socialement, les quartiers d'Errachidia, à l'instar des quartiers étudiés (Targa Jdida et Centre-ville), sont pour la majorité des quartiers populaires.

Ce qui différencie aussi Rabat d'Errachidia est la présence visible dans cette dernière d'une population amazighe/amazighophone issue des ksours de la région.

Il est à signaler qu'à l'intérieur même de Rabat existent des inégalités à toutes les échelles entre les quartiers où on distingue entre les quartiers populaires (cf. Akkari) et les quartiers aisés (cf. Hay Ryad).

\title{
1.4.2.1. Les enseignes relevées à Rabat
}

Les enseignes collectées à Rabat, dans les quartiers Hay Ryad et Akkari, s'élèvent à 158 photos réparties comme suit : 83 à Hay Ryad et 75 à Akkari.

\section{- Hay Ryad}

Le classement des langues de l'environnement graphique de Hay Ryad, selon notre corpus, révèle que le français est grandement visible dans ce quartier avec 59\% des enseignes occupées, suivi de loin du bilinguisme arabe-français avec $20 \%$. Cependant, les enseignes trilingues où le français est présent à côté des deux langues officielles de l'Etat ne détiennent qu'un faible taux de $6 \%$.

Par ailleurs, nous avons constaté que l'emploi monolingue de l'anglais sur les enseignes de Hay Ryad a atteint $12 \%$, ce qui dépasse de loin le trilinguisme institutionnel arabe-amazigh-français.

\begin{abstract}
- Akkari
Au quartier Akkari, la configuration change. Le français perd sa position de première langue d'affichage acquise à Hay Ryad. Désormais, il est relégué à la troisième position avec seulement $23 \%$ des enseignes. La première et la deuxième place sont occupées respectivement par les enseignes bilingues arabe-français avec $36 \%$ et les enseignes monolingues arabes avec $24 \%$. Cependant, il y a lieu de signaler que le français et l'arabe sont presque à égalité dans les enseignes de ce lieu d'enquête.

Toutefois, le trio arabe-amazigh-français combiné est encore à la traine dans ce quartier populaire avec seulement $10 \%$ de l'emploi dans les enseignes. Quant à l'anglais, il n'est présent que dans $4 \%$ des enseignes.
\end{abstract}




\subsubsection{Les enseignes relevées à Errachidia}

Dans les deux quartiers d'Errachidia, Centre ville et Targa Jdida, l'emploi bilingue arabe-français vient en tête d'affiche. Il est présent dans $37 \%$ des enseignes, suivi, en deuxième position, de l'usage trilingue arabe-amazighfrançais avec un taux de $30 \%$ des enseignes.

A noter qu'il y a dix ans de cela, le tamazight était absent des enseignes tant publiques que privées de la ville d'Errachidia (Khiri 2009). La troisième position revient à l'emploi de l'arabe seul dans les enseignes avec $15 \%$. Quant au français seul, il n'est mobilisé que dans $13 \%$ des enseignes. Le bilinguisme arabe-amazigh n'est visible que dans $4 \%$. L'anglais et l'espagnol sont très peu représentés.

\subsubsection{Observations sur l'usage des langues dans la signalétique selon les lieux d'enquête}

L'observation des langues employées dans la signalétique selon les lieux d'enquête montre qu'à Hay Ryad, le choix des langues des enseignes est conditionné par plusieurs facteurs socio-économiques et identitaires.

La prédominance du français dans ce quartier chic de Rabat constitué «majoritairement de cadres supérieurs (de l'administration, des banques, du commerce et de l'industrie) et de hauts fonctionnaires » (Serhir $2017: 23$ ), est le reflet des choix de la communauté qui y réside et qui se considère comme une élite francophone ayant fréquenté «les écoles françaises, s'exprimant majoritairement en français, y compris en famille, dans le quotidien et dont les enfants apprennent le français dès leur jeune âge et qui ont pour ainsi dire deux langues maternelles ; parfois, ils maitrisent plus le français que l'arabe » (Messaoudi \& Mzioud 2012 : 177).

Hay Ryad abrite en plus, de grandes administrations nationales et régionales, des établissements publics européens à l'instar de la Délégation européenne et la Banque européenne d'investissement. Ce quartier est aussi le lieu de prédilection de plusieurs marques commerciales franchisées.

La langue française, qui est très largement utilisée dans la signalétique de Hay Ryad, a reculé dans les enseignes du quartier Akkari et dans celles des deux quartiers d'Errachidia pour arriver respectivement en $3^{\text {ème }}$ et $4^{\text {ème }}$ position.

Dans ces quartiers, les enseignes bilingues arabe-français viennent en première position, ce qui est ordinaire pour un pays comme le Maroc qui a opté pour ce bilinguisme de fait, depuis l'indépendance et notamment depuis 
l'arabisation de plusieurs secteurs socioéconomiques tout en maintenant, le français comme première langue étrangère privilégiée, assurant à la fois une fonction élitaire et utilitaire (Messaoudi : 2010). Quant à l'emploi de l'arabe, il détient le même taux d'usage de $24 \%$.

La seule différence entre les quatre sites enquêtés est la présence remarquable, en deuxième position après les enseignes bilingues arabe-français, de l'amazigh à côté de l'arabe et du français dans les enseignes des deux quartiers d'Errachidia, ceci s'explique par la présence d'une communauté amazighe importante à Errachidia.

Qu'en est-il du choix des langues des enseignes selon les secteurs : publics et privés?

\subsubsection{Les enseignes des secteurs publics et privés}

Il y a lieu d'opérer un classement distinguant les enseignes en publiques et privées. Le corpus des photos des enseignes collectées dans les sites d'enquête comprend 46 enseignes du secteur public et 232 enseignes du secteur privé.

\subsubsection{Les enseignes du secteur public}

Les enseignes du secteur public les plus utilisées dans les quartiers observés à Rabat et à Errachidia sont trilingues arabe-amazigh-français. Ce trilinguisme est observé dans la majeure partie des administrations et des offices publics. Par ailleurs, nous avons constaté que le bilinguisme arabe-français est encore en vigueur dans les anciennes enseignes de quelques communes, des noms de rues et des panneaux de signalisation et d'indication routière en souvenir des années où l'amazigh n'est pas encore officialisé.

Ainsi l'environnement linguistique des quartiers des deux villes connait la présence de l'amazigh depuis l'adoption de la constitution de 2011 qui le reconnait comme une deuxième langue officielle à côté de l'arabe. Il est aussi à noter que les enseignes monolingues arabes des établissements scolaires publics ont été remplacées par des enseignes bilingues arabe-amazigh conformément à la nouvelle tendance de la reconnaissance de l'amazigh comme langue officielle à côté de l'arabe ${ }^{(8)}$.

(8) La Constitution marocaine adoptée en 2011 considère l'arabe et l'amazigh comme deux langues officielles de l’État (sans préciser ni de quel « arabe » ni de quel « amazigh » il s'agit). 
La présence de l'amazigh consigné en tifinagh( ${ }^{(9)}$ dans les enseignes publiques n'a pas, jusqu'à présent, de fonction informationnelle ; son emploi demeure symbolique. C'est une forme de reconnaissance par l'Etat des droits culturels amazighs ${ }^{(10)}$.

Nous pouvons augurer que d'ici quelques années le modèle d'enseigne publique marocaine ne serait que trilingue arabe-amazigh-français.

Cette forme d'enseignes publiques trilingues sera-t-elle maintenue dans le secteur privé ?

\subsubsection{Les enseignes du secteur privé}

Les enseignes du secteur privé collectées concernent les banques et assurances; les cabinets privés ; les magasins ; les hôtels, cafés et restaurants ; les établissements scolaires privés ; les pharmacies et le secteur tertiaire des services de prestation.

Une vue d'ensemble sur les enseignes de ce secteur montre que le bilinguisme arabe-français et le monolinguisme français sont prédominants. Ils s'accaparent à égalité de plus de $68 \%$ des enseignes privées. L'emploi de l'arabe seul vient en troisième position avec seulement $13 \%$. Par contre, le modèle de l'enseigne publique n'est représenté que sur 9\% des enseignes privées; les préoccupations des pouvoirs publics ne semblent pas intéresser les acteurs du secteur privé. Quant à l'emploi seul de l'anglais, il n'est attesté que sur environ $6 \%$ des enseignes privées, ce qui montre que la demande est encore timide dans ce sens.

Cela dit, ce constat général quant à l'emploi des langues dans les enseignes privées des quartiers étudiés reste à vérifier puisque chaque site d'enquête présente des particularités qui lui sont propres.

A Hay Ryad, le français est utilisé seul sur plus de $62 \%$ des enseignes au moment où d'autres enseignes affichent le bilinguisme arabe-français et le monolinguisme anglais.

(9) Graphie amazighe.

(10) Cette tendance est également observée dans quelques enseignes publiques où l'amazigh est utilisé à côté de français comme c'est le cas sur les panneaux de signalisation routière dans les nouveaux tronçons des autoroutes ouverts à la circulation il y a quelques années. (Tronçon pont Mohamed VI et autoroute Casablanca - Béni Mellal). 
A Akkari et dans les deux quartiers d'Errachidia, le bilinguisme arabefrançais est largement utilisé sur plus de $40 \%$ des enseignes privées. Cependant, l'emploi de l'arabe seul vient en deuxième position à Akkari, mais il occupe la troisième place à Targa Jdida et au Centre ville à Errachidia ; la deuxième position étant occupée à Errachidia par le trilinguisme arabe-amazigh-français.

Pour récapituler, nous pouvons dire que le monolinguisme français fait légion dans les enseignes de Hay Ryad, ce qui confirme la particularité socioéconomique de ce quartier. Par contre le bilinguisme arabe-français est confirmé à Akkari, à Traga Jdida et au Centre ville d'Errachidia. Par ailleurs, le paysage linguistique d'Errachidia libère de plus en plus d'espace pour le trilinguisme arabe-amazigh-français. L'emploi de l'arabe seul se maintient dans les enseignes des magasins et des échoppes des services de prestations dans les quartiers populaires. Chaque domaine d'activité pourrait-il donc avoir une certaine préférence linguistique dans la conception des enseignes afférentes?

\subsubsection{Les langues des enseignes selon les domaines d'activités}

Nous avons constaté que les variétés linguistiques employées dans les enseignes des banques et assurances (dans les quatre sites d'enquête) sont bilingues arabe-français en application d'une pratique bilingue en usage depuis longtemps par les banques marocaines. Toutefois, il y a lieu de signaler que les Banques participatives (qu'on appelle communément les banques "islamiques") sont plutôt pour un bilinguisme arabe-anglais comme cela se fait dans les pays du Golf d'où proviennent le concept et une grande partie des capitaux de ces banques.

Pour les magasins de vente des produits de consommation (hors alimentation), ils arborent à Hay Ryad des enseignes unilingues français au vu de la particularité de sa population cultivée et aisée, et notamment en rapport avec les produits de haute gamme offerts par ces magasins et qui ne sont à la portée que des personnes aisées.

Par contre, c'est le bilinguisme arabe-français qui occupe la place d'honneur dans les enseignes des magasins de Akkari, ceci est du à son statut de quartier populaire.

Quant aux magasins des deux quartiers d'Errachidia, ils ont une nette préférence pour les enseignes trilingues arabe-amazighe-français en premier lieu et les enseignes unilingues arabes en deuxième lieu, ce qui reflète les attitudes de la population d'Errachidia vis-à-vis des langues entre une tranche conservatrice 
pour qui l'arabe est «la seule langue standard des Marocains » et une autre tranche plus tolérante qui reconnait en toute ouverture d'esprit toutes les autres variétés linguistiques, notamment l'amazigh.

Notons également, qu'il y a des détenteurs de magasins à Errachidia qui ne parlent pas l'amazigh, mais qui ont remplacé leurs anciennes enseignes monolingues arabes ou bilingues arabe-français par des enseignes trilingues arabe-amazigh-français pour attirer la clientèle amazighophone.

En ce qui concerne le domaine intitulé « cabinets privés », nous les avons scindés en deux types : les cabinets des avocats, des médecins et les bureaux des adouls $^{(11)}$. Les premiers optent pour les enseignes bilingues, par contre les seconds, dont le métier est lié à l'organisation des transactions entre les gens selon la loi musulmane, se contentent des enseignes unilingues en arabe.

Le domaine qui connait un accord tacite quant aux variétés linguistiques à utiliser sur les enseignes, dans les quatre sites d'enquête, est le domaine « Hôtels, cafés, restaurants ». Les décideurs de ce secteur sont presque unanimes à employer le français seul sur leurs enseignes.

L'emploi du français peut être justifié par le souci d'attirer des touristes français. Mais pourquoi une grande partie des cafés tout au long de l'avenue de Targa Jdida (Errachidia), zone non touristique, affichent-ils des enseignes unilingues en français? Ne serait-ce pas pour notifier aux passants que le service est de bonne qualité ? Le passant, pour sa part, ne le perçoit-il pas comme un café prestigieux?

En fait, le choix des langues des enseignes n'est pas fortuit. Il est lié au type de quartier (aisé/populaire), au niveau de sa population (cultivée, riche, pauvre), de sa composition (arabe, amazighe, étrangère), des langues de ses groupes linguistiques, de la qualité et/ou du coût du service ou de la marchandise offerts. Il est en rapport également avec son rôle informationnel, commercial ou symbolique, puisque l'emploi d'une telle langue peut séduire un type de clientèle qui parle cette langue, ou qui la considère comme prestigieuse, valorisante ou identitaire.

Pour clore ce premier axe, il convient de noter que le dénominateur commun entre ces lieux d'enquête à Rabat et à Errachidia est le non recours à l'usage de la darija dans la signalétique et les affiches non publicitaires.

(11) Pluriel d'adel qui est un officier public chargé de dresser les actes relatifs au statut personnel ainsi que concurremment, avec les notaires, certains autres actes de la vie civile. 
Dans le $2^{\mathrm{e}}$ axe, qui suit, nous nous intéresserons aux langues des enseignes dans la publicité.

\section{Les langues des enseignes dans la publicité}

La publicité est un genre de communication particulier qui est caractérisé par trois éléments : le message linguistique, le message iconographique et le logo.

Sur le plan linguistique, le message publicitaire comprend le slogan (d'accroche) qui est souvent une formule courte, destinée à être aisément mémorisée et répétée par un nombre illimité de locuteurs. La seconde partie du slogan, dite d'assise, souvent placée en bas du placard publicitaire, en petites lettres, apporte les indications pratiques et les informations complémentaires.

Le contenu verbal de la publicité remplit en fait plusieurs fonctions : informer, séduire, argumenter, etc. Pour atteindre le consommateur, tous les moyens sont mobilisés comme la taille des caractères, de l'affiche, la disposition des caractères, la place du texte publicitaire, les variétés linguistiques sollicitées, etc.

La structure du contenu verbal, le plus souvent constitué d'une formule courte, est rythmique, répétitive, binaire (rimes, nombre de syllabes, etc.) ressemble à celle d'un proverbe.

Voici un exemple à titre illustratif: ذواق وغلاق (đuwwàq w Rallàq) « gourmet et gourmand ». Cette formule est inspirée d'un adage en darija qui est đuwwàq maši Rallàq « gourmet pas gourmand » (voir figure ci-dessous)

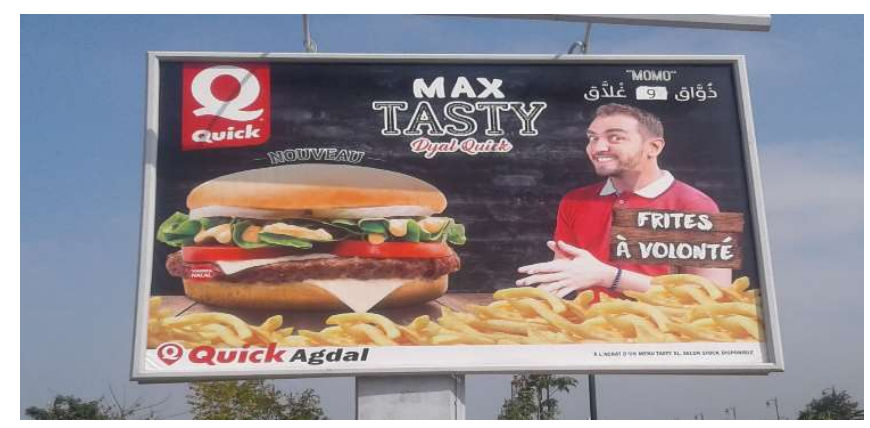

Fig. 2 
Après avoir défini succinctement le message publicitaire qui est à distinguer de celui de la signalétique urbaine, nous allons examiner le corpus en essayant de fournir des éléments de réponse aux questions suivantes : quelles sont les variétés linguistiques utilisées en contexte urbain dans le message publicitaire au Maroc ? Que signifie ce choix linguistique ? Sur quelle politique linguistique s'appuie-til ? Quelle est la caractéristique du message publicitaire : celle de l'unicité du code linguistique ou bien celle de l'hybridité ? Le message publicitaire s'adaptet-il au type de territoire (par exemple, quartier huppé et quartier populaire) ou bien est-il le même dans les deux espaces considérés ?

Les résultats de l'analyse nous permettront de dégager en filigrane, la tendance de la politique linguistique dans le domaine de la publicité pour voir si elle est en faveur de telle ou telle variété linguistique ou en faveur du mélange de langues.

\subsection{Variétés linguistiques utilisées dans les affiches publicitaires selon les} sites d'enquête

Les affiches publicitaires retenues sont au nombre de 29. D'après notre enquête, l'usage combiné de l'arabe (standard) et de Darija (arabe dialectal) vient en tête avec $35 \%$ des planches publicitaires avec une primauté d'usage pour Akkari, quartier populaire.

L'usage d'une seule langue : l'arabe (standard) seul ou le français seul pour Ryad, quartier huppé.

Aucun usage de l'amazighe dans les affiches publicitaires récoltées ; même pas à Errachidia où la signalétique officielle et les panneaux institutionnels en font pourtant usage.

Le graphique suivant illustre le taux d'usage des langues dans les enseignes des sites enquêtés. La darija est la variété linguistique la plus employée dans les messages publicitaires. 


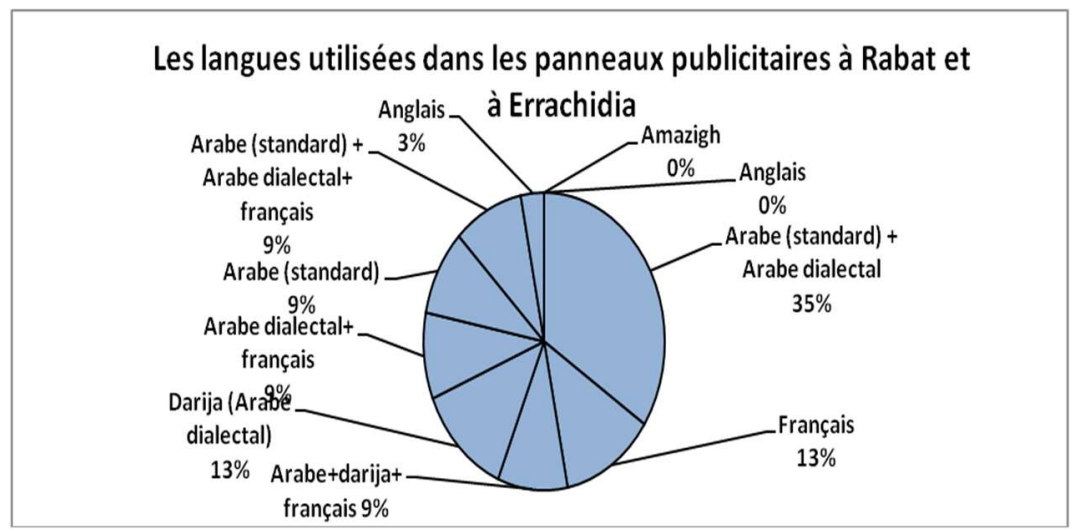

Fig. 3

2.1.1. Variétés linguistiques utilisées dans les affiches publicitaires selon le type d'activité

Dans le corpus collecté, le domaine des Télécoms représente plus de $65 \%$ des enseignes, celui des finances plus de $20 \%$ et celui du bâtiment environ $3 \%$. D'autres domaines (restauration, poste, etc.) sont de l'ordre de 10\%. La variété la plus utilisée est sans conteste la darija seule ou à côté d'autres langues à raison de $68 \%$ pour les Télécoms, de $100 \%$ pour les banques, des sociétés du crédit et du microcrédit. Le secteur du bâtiment fait aussi usage de la darija notamment dans la promotion de l'habitat social à cout modéré. En revanche, la darija n'est utilisée seul que dans près de $10 \%$ des enseignes tous secteurs confondus.

\subsection{Quelques exemples du message linguistique publicitaire}

\subsubsection{Message monolingue : unicité du code linguistique}

Les messages monolingues usent de l'arabe, du français, ou de la darija.

Voici quelques exemples puisés du corpus :

\subsubsection{En arabe (standard) seul}

- Office National des Chemins de Fer (ONCF)

$$
\text { عيشوا أجمل الأحاسيس على متن قطاراتنا، على خطوطنا تظهر معالم الحضارة ONCF }
$$


ONCF (çišu 'ajmal al'aHasis çala matni qiTaratina, çala xuTuTina tađharu maçalim lHaDara). « ONCF, vivez de plus belles sensations à bord de nos trains, sur nos lignes apparaissent les contours de la civilisation »

\section{- Assurances}

$$
\begin{aligned}
& \text { سند أوطو (sanad UTu) «Sanad autou» } \\
& \text { (çard çala maqasikum) « offre sur votre mesure » } \\
& \text { جر السيارة (jerr ssiyara) 《remorquer la voiture » } \\
& \text { سيارة بديلة (siyara badila) 《 voiture de remplacement» } \\
& \text { تعويض سريع (taçwiD sariç) 《indemnisation rapide 》 } \\
& \text { وأزيد من } 180 \text { وكالة في خدمتم (wa azyad min } 180 \text { wukala fi xidmatikum) « et plus } \\
& \text { de180 agences à votre service »" } \\
& \text { (fi kulli 'anHa' lmaRrib) 《dans tout le Maroc» }
\end{aligned}
$$

\subsubsection{En français seul}

\section{- ONCF}

1 départ toutes les 2 heures, joindre le rapide à l'agréable

- Opérateur Telecom
Je suis $100 \%$ autonome
Cadeau du vendredi
Cinéday
Recharge Flex
Smart Solde
Payer ma facture
Changer mon forfait
Changer mon numéro

\subsubsection{En arabe dialectal seul et en graphie arabe}

فرمة عين فلوسك توصل فالحين (firamšst çin flusk tusl f lHin) «En un clin d'œil, ton argent arrivera sur le champs »

يسهل حياتك، يدفع الأجرة حتى تدوز خلصتك (yəshl hyatk, yədfç lujra Htta dduz xəlstk) «Il te facilite la vie, paye ton salaire jusqu'à ce que ta paie soit versée »

- Opérateur Telecom

(استفيدوا من (stafidu min) «bénéficiez de... » 
10 Go (10 Go iDafiya) «10 Go supplémentaires »

مكونيكتي ديما أكثر (mkunikTi dima 'aktar) 《 connecté toujours davantage »

فوز بالمموز (fuz blhmuz) 《gagne de bonnes occasions »

- Immobilier

عيط تربح (عyT trbH) «Appelle (par téléphone) et tu gagnes »

شري دارك و ربح فالحين العديد من الهدايا (šri dark wrbH flHin leadid mn lhadaya)

" achète ta maison et gagne sur le champs plusieurs cadeaux "

(šuqaq 3 aw 2 Ruraf za'id Salun) « appartements : 3 ou 2 pièces + salon »

\subsubsection{En arabe dialectal seul et en graphie latine}

fouz belhmouz « gagne des occasions »

fremšet çin, flusk tuSel $f l$ Hin «En un clin d'œil, ton argent arrivera à temps »

Yum wra yum, er-rušarj fabur kull yum « Jour après jour, la recharge est gratuite chaque jour »

Dar lik u Dar lwalidik « une maison pour toi, une autre pour tes parents »

\subsubsection{En arabe standard et dialectal}

سند بالحل ديما يجيك (sanad blHl dima yijik) «Sanad t'apporte toujours des solutions "

سند للتأمين بجانبكم كل يوم (sanad litta'amin bijanibikum kulla yawm) «Sanad assurance à vos côtés tous les jours »

سند أوطو $($ sanad uTu) «Sanad autou»

(çard çala maqasikum) « offre sur votre mesure »

(jerr ssiyara) « remorquer la voiture »

سيارة بديلة (siyara badila) 《 voiture de remplacement 》

تعويض سريع (taçwiD sariç) «indemnisation rapide »

- Micro crédit

بغيتي تمويل يناسبك و تطور مشروعك، البركة هي عنوانك (bRiti tamwil ynasbk wtวTwwar mšruçk lbaraka hiya çnwank) « Tu veux un financement qui te convient et qui développe ton projet, lbaraka est ton adresse »

(استفيدوا من خبرة 20 سنة ('istafidu min xibrat çišrin sana) "profitez d'une experience de 20 ans » 
(fi tamwil lquruD SSuRra) «dans le domaine du financement des microcrédits »

\subsubsection{Message plurilingue en une ou plusieurs graphies : hybridité du code} linguistique

Quelques exemples tirés du corpus serviront à illustrer les types d'hybridité et de mélange de codes des messages, suivant différents secteurs d'activité. Notons que la numérotation des types est utilisée pour le classement des messages extraits du corpus et ne comporte aucune valeur appréciative.

- Télécom

Type 1 : Darija + français (une seule graphie : latine)

LMOUHIM j'ai un abonnement XXX « l'essentiel», j'ai un abonnement $\mathrm{XXX}$

Type 2 : Français (en deux graphies : arabe et latine)

$$
\text { فوريف PRO « forfait » PRO }
$$

Type 3 : Darija (en graphie latine) + français (graphie latine) + darija (graphie arabe)

MA! TKHEMEMCH « ne te fais pas de soucis »

Smartphone +3 mois Illimités de...

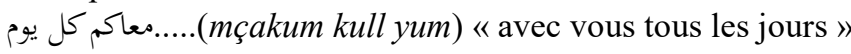

- Poste

Type 4 : darija (en graphie arabe et latine) + français (graphie latine) + arabe standard (graphie arabe)

كلشي اليوم غيبغي يسيفط الفلوس مع (kulši Ray bRi yisifT lflus mça) « Aujourd'hui, tout le monde voudra envoyer de l'argent avec... »

Mandati

(taHwil l'amwal) « transfert d'argent »

2000 (alfayn dirham lilfawz kul yawm) «2000 dirhams à gagner chaque jour $»$

- Restauration

Type 5 : anglais (graphie latine) + darija (graphie arabe) + français (graphie latine)

Fast food ذواق وغلاق (dawwaq w Rallaq) 《 gourmet et gourmand » 
Max tasty

Frites à volonté

\subsubsection{Hybridité et néologie lexicale}

On peut citer un premier exemple, à partir de la planche publicitaire cidessous. Il s'agit du mot Inzeglable qui est formé à partir du préfixe privatif français « in »+ le radical du lexème zgel (en darija signifie « rater ») + le suffixe français « able ». Ce néologisme hybride aurait pour synonyme en français le mot « inratable ».

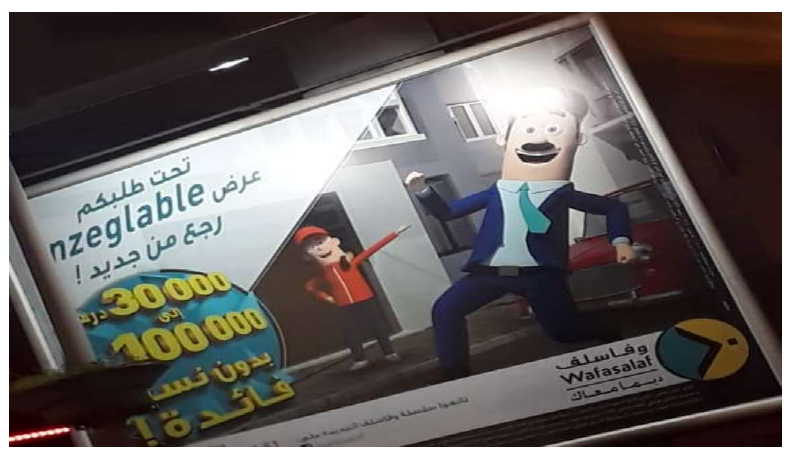

Fig. 4

Un deuxième exemple peut être celui du mot valise Les Imposta7il, mot formé à partir du français «impo » (de «impossible ») + de l'arabe sta7il (de mousta7il («impossible») comme cela ressort de la planche publicitaire cidessous : 


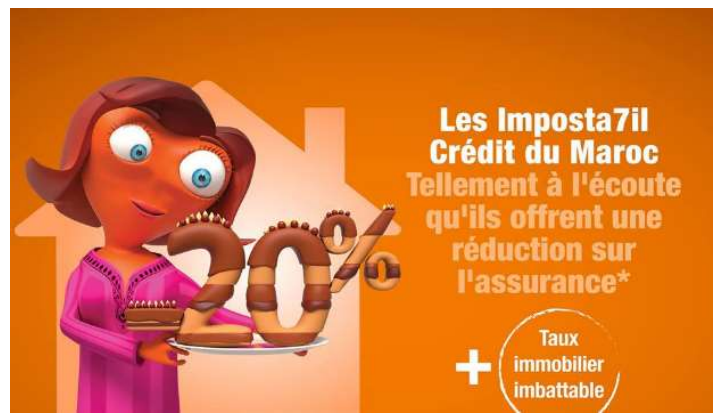

Fig. 5

\subsubsection{Observations sur le message publicitaire et l'unicité ou l'hybridité des} codes

Le contact de variétés linguistiques a favorisé l'émergence de «parlers hybrides», faits de mélanges et obéissant à une sorte de créolisation comme formes d'appropriation des_variétés linguistiques circulantes dans une société « composite » (PASCON 1971), influencée à la fois par le local et le global (glocal) via Internet (depuis la fin du 20e siècle).

La publicité est à l'image des interactions verbales où la darija et les parlers hybrides occupent une place prépondérante. L'éventail des usages linguistiques médiatiques s'est considérablement élargi, transformant de fait la hiérarchie traditionnelle des variétés linguistiques au Maroc, telle qu'elle était encore présentée jusqu'à une époque récente et qui comprenait l'arabe standard et le français comme usages de l'écrit et de l'oralité formelle et la darija et l'amazighe comme usages de l'oralité (Boukous 1995, Messaoudi 2002).

\subsubsection{Quelle politique linguistique dans la publicité? Quelles tendances?}

Les situations linguistiques de par le monde oscillent entre différentes tendances: celle interventionniste et fortement centralisatrice orientée par la Constitution et les textes officiels comme les bulletins et les journaux officiels ; et l'autre est libérale et se pratique par défaut. Le manque de réglementation n'implique pas l'absence d'une politique. Il s'agit d'une politique qui repose à la fois sur le laissez faire et sur l'autorégulation «spontanée » qui en découle. 


\subsubsection{Au Maroc: quelle politique linguistique dans la publicité ?}

La politique linguistique marocaine prend appui sur ces deux tendances. Elle allie une législation linguistique unificatrice à l'échelle nationale, qui se traduit par la Constitution du Royaume du Maroc, mais elle est en même temps libérale, puisque l'usage des autres variétés linguistiques n'est pas réglementé. Il n'est pas prescrit mais il n'est pas non plus proscrit. La politique linguistique de la publicité est à l'image de celle du pays: d'un côté, il existe des usages officiels ; de l'autre côté, des usages informels et des mélanges reflétant les pratiques des locuteurs. La publicité s'inspire des pratiques linguistiques des locuteurs, notamment celles populaires.

L'emploi de la darija dans la publicité n'est plus réservé aux locuteurs des couches défavorisées (analphabètes, milieux ruraux, rurbains, etc.), mais s'adresse également aux couches moyennes en milieu urbain et même aux couches supérieures.

De plus, les jeux de mots, les créations à caractère ludique sont utilisées sciemment pour attirer l'attention des acteurs sociaux et influencer leur choix pour l'acquisition de tel ou tel produit ou pour l'adhésion à telle ou telle activité. Ce qui explique le choix linguistique des publicitaires pour des messages en darija et dans des mélanges pour la téléphonie mobile, les services, les transports, etc. et non plus seulement pour des produits de lessive ou des huiles alimentaires.

Ceci étant, les représentations des locuteurs vis-à-vis des variétés linguistiques ne sont pas toujours en conformité avec les pratiques (Meskine \& De Ruiter 2016) et malgré cela, les publicitaires adossent leurs productions aux pratiques orales prédominantes dans la société, celles de la darija. L'on peut se demander à quelles fins sont employées les variétés linguistiques et quelles valeurs ont à transmettre.

\subsubsection{Usages linguistiques dans la publicité au Maroc: transmission de quelles valeurs?}

Il est évident que les messages publicitaires transmettent des valeurs selon la cible visée par le slogan et selon la variété linguistique utilisée. S'agit-il d'une recherche d'une tentative de rapprochement avec le destinataire ? S'agit-il de conservatisme? S'agit-il de modernisme?

L'usage de telle ou telle variété linguistique dans les enseignes publicitaires n'est pas fortuit et induit, en filigrane, une représentation implicite de la valeur 
symbolique de tel ou tel usage linguistique ; par exemple, dans le message : عيشوا (12). أجمل الأحاسيس على متن قطاراتنا، على خطوطنا تظهر معالم الحضارة

Par ce message en arabe standard, l'émetteur vise à répondre au besoin du consommateur marocain lettré en arabe, soucieux d'utiliser la langue prestigieuse et de préserver son identité. En revanche, le français peut être préféré par un autre publicitaire, s'adressant à un locuteur marocain qui voit dans cette variété non seulement du prestige mais aussi un aspect plus pratique. Ce locuteur ne peut être attiré que par un slogan pratique et moderne, du genre : «Un départ toutes les deux heures, joindre le rapide à l'agréable ».

Ceci étant, il convient de noter, par ailleurs, que toutes les langues en usage dans les enseignes véhiculent des valeurs marchandes : c'est l'essence de tout message publicitaire. Et ce, sans perdre de vue les valeurs marchandes des langues et le pouvoir économique de telle ou telle langue (Grin 2009, Messaoudi 2012).

La darija, à usage prédominant dans la publicité marocaine, obéit à ce principe et vise à s'adresser à la majorité des locuteurs et locutrices mais elle peut pécher par deux défauts : d'un côté, la créativité lexicale à caractère hybride n'est pas d'emblée comprise par le destinataire ${ }^{(13)}$ et d'un autre côté, elle peut être porteuse de stéréotypes ; par exemple, à caractère sexiste comme dans le message suivant :-ما عمرها ما حشماتني هو وجهي، زيت (Tyabi huwwa wjhi, ziyt ....Ma çmmarha ma Hšmatni) «ma cuisine est ma face, l'huile ....ne m’a jamais éhontée ».

\section{Conclusion}

Levi-Strauss (1974 : 74) considérait «Le langage à la fois comme le fait culturel par excellence et celui par l'intermédiaire duquel toutes les formes de la vie sociale s'établissent et se perpétuent ».

Le jeu des variétés linguistiques dans l'affichage public et privé mais aussi dans la publicité est à l'image de la société marocaine, à la fois « composite » et « en mutation » sous le poids de la « glocalisation».

Les résultats de l'enquête ont montré que l'usage du trilinguisme institutionnel arabe standard-amazigh-français est l'apanage de l'affichage officiel public (ministères, administrations, délégations, établissements scolaires,

(12) Pour la transcription et la traduction, voir 2.2.1.1.ici même.

(13) Voir 2.2.5, ici même. 
hôpitaux, etc.) ; en revanche, le secteur du privé offre une panoplie d'usages et se permet une plus grande liberté dans le choix des variétés linguistiques.

Mais c'est surtout le domaine de la publicité, notamment dans le secteur des Télécom, qu'il y a le plus grand nombre de mélanges et où la darija est prédominante. Une question importante en découle : celle du passage à l'écrit.

Selon Jack Goody (1979: 46-47), auteur d'une étude sur l'impact de l'écriture et le lien qui existe entre le mode de pensée et les moyens de pensée : «tout changement dans le système de communications a nécessairement d'importants effets sur le contenu transmis ».

L'écrit agit donc. Et faire muter la darija, en la transposant à l'écrit peut avoir des incidences concrètes sur la société et les institutions autour desquelles elle s'organise. Ecrire la langue populaire revient à lui donner une consistance, un corps amenant à l'employer. La société marocaine est-elle prête pour cette mutation?

Des études et des enquêtes sur les usages des variétés linguistiques et leur portée sont à effectuer pour fournir des éléments de réponse aux questions formulées.

\section{BIBLIOGRAPHIE}

BOUKOUS, Ahmed. 1995. Société, langues et cultures au Maroc. Enjeux symboliques. Rabat, Publications de la Faculté des Lettres et des sciences humaines de Rabat.

BULOT, Thierry. 2011. Sociolinguistique urbaine, Linguistic Landscape Studies et scripturalité : entre convergence(s) et divergence(s). Cahiers de Linguistique 37 (1), pp. 5-15.

$<$ https://halshs.archives-ouvertes.fr/halshs-00654813> [19 juillet 2019].

BULOT, Thierry et MESSAOUDI, Leila. 2003. Sociolinguistique urbaine. Frontières et territoires. Cortil-Wodon, Editions modulaires européennes.

CALVET, Jean Louis. 1994. Les voix de la ville : Introduction à la sociolinguistique urbaine. Essais, Essais.

GOODY, Jack. 1979. La Raison graphique. Paris, les Éditions de Minuit. 
GORTER, Durk. 2006. Introduction: «The Study of the Linguistic Landscape as New Approach to Multilinguism». In: Linguistic Landscape as New Approach to Multilingualism. Clevedon, Multilingual Matters Ltd., pp 1-7.

KHIRI Mustapha. 2009. Parler arabe d'Errachidia des ksour à la ville. Thèse de Doctorat national, Faculté des Lettres et des Sciences humaines de Kénitra.

KHIRI Mustapha. 2017. «Le parler beldi de la ville d'Errachidia : langue, espace et identité ». Langues, cultures et sociétés 3 (1), Rabat, CNRST- IMIST, pp. 100-112.

$<$ https://revues.imist.ma/index.php?journal=LCS $>$ [19 juillet 2019].

LANDRY, Rodrigue \& BOURHIS, Richard Y. 1997. «Linguistic landscape and ethnolinguistic vitality: An empirical study». In: Journal of Language and Social Psychology, 16 (1), pp. 23-49.

LEVI-STRAUSS, Claude. 1974. Anthropologie structurale. Paris, Plon.

MESKINE, Driss \& DE REUTER, Jan Jaap. 2016. «Les jeunes Marocains s'expriment: le marché des langues du Maroc en mutation ». Dans: Francisco Moscoso García \& Adil Moustaoui Sghir (eds). Identidad y conciencia lingüistíca. Madrid, Ediciones Universidad Autónoma de Madrid, pp. 21- 46.

MESSAOUDI, Leila. 2002. «L'aménagement linguistique au Maroc ». Dans : Khatibi (dir.) BESM. Rabat, Okad.

MESSAOUDI, Leila. 2010. « La langue française au Maroc. Fonctions élitaire ou utilitaire ?». Dans : Philippe Blanchet, Pierre Martinez (dir.). Pratiques innovantes du plurilinguisme: Emergence et prise en compte en situations francophones. Paris, Editions archives contemporaines AUF.

MESSAOUDI, Leila. 2014. «Plurilinguisme et pouvoir économique au Maroc. Quelle place pour la langue française?». Dans : Romain Colonna (éd.). Les locuteurs et les langues: pouvoirs, non pouvoirs et contre-pouvoirs. Limoges, Lambert-Lucas.

MESSAOUDI, Leila \& MZIOUD, Hajar. 2012. «Le plurilinguisme urbain: marquages et discrimination des espaces à Rabat (Maroc)». Dans : Ségrégation, normes et discriminations. Sociolinguistique urbaine et migrance. Bruxelles, EME et intercommunications, pp. $177-213$

PASCON Paul. 1971. «La formation de la société marocaine ». Dans : BESM $121-122$, pp. 1-25. 